\title{
ANALYSE LONGITUDINALE DES FACTEURS PERSONNELS ET PROFESSIONNELS ASSOCIÉS AU TURNOVER PARMI LES SOIGNANTS
}

\author{
Madelaine Estryn-Behar et al.
}

A.R.S.I. | Recherche en soins infirmiers

$2010 / 4-N^{\circ} 103$

pages 29 à 45

ISSN 0297-2964

Article disponible en ligne à l'adresse:

http://www.cairn.info/revue-recherche-en-soins-infirmiers-2010-4-page-29.htm

Pour citer cet article :

Estryn-Behar Madelaine et al., « Analyse longitudinale des facteurs personnels et professionnels associés au turnover parmi les soignants ",

Recherche en soins infirmiers, 2010/4 №103, p. 29-45.

Distribution électronique Cairn.info pour A.R.S.I..

(C) A.R.S.I.. Tous droits réservés pour tous pays.

La reproduction ou représentation de cet article, notamment par photocopie, n'est autorisée que dans les limites des conditions générales d'utilisation du site ou, le cas échéant, des conditions générales de la licence souscrite par votre établissement. Toute autre reproduction ou représentation, en tout ou partie, sous quelque forme et de quelque manière que ce soit, est interdite sauf accord préalable et écrit de l'éditeur, en dehors des cas prévus par la législation en vigueur en France. II est précisé que son stockage dans une base de données est également interdit. 


\section{ANALYSE LONGITUDINALE DES FACTEURS PERSONNELS ET PROFESSIONNELS ASSOCIÉS AUTURNOVER PARMI LES SOIGNANTS}

Madelaine ESTRYN-BEHAR,

Médecin du travail, épidémiologiste, ergonome, Paris, France

Béatrice I.J.M.VAN DER HEIJDEN,

Professeur de management stratégique des ressources humaines, Institute for Management Research, Radboud University Nijmegen, et avec la participation des universités Open University of the Netherlands et l'University of Twente, Pays Bas

Clémentine FRY,

Statisticienne, Paris, France

Hans-Martin HASSELHORN, Professeur de médecine du travail, Université de Wuppertal,Allemagne et le groupe NEXT (Europeen Nurses'Early Exit Study), Wuppertal,Allemagne

\section{Nous remercions les auteurs et l'éditeur de la revue « Nursing Research » de nous avoir permis de publier cet article dans Recherche en soins infirmiers.}

Cet article a été publié sous le titre « Longitudinal Analysis of Personal and Work-Related Factors Associated with Turnover among Nurses ॥, in Nursing Research, Spec Mat: NNR, 2010; 59 (3): I56-I77. II a été traduit en français par un des auteurs, Madeleine Estryn-Béhar.

\begin{tabular}{|l} 
R E S U M E \\
personnels et professionnels associés \\
au turnover parmi les soignants \\
Contexte: Alors que de nombreuses études \\
ont mis en évidence les relations entre les fac- \\
teurs personnels et professionnels associées à \\
la recherche d'emploi et au turnover, il y a un \\
manque de design longitudinaux qui testent \\
cette relation de façon empirique. \\
Objectifs: Cette étude longitudinale examine les \\
facteurs déterminants spécifiques qui différencient \\
ceux que l'on appelle les «restants» et les «par- \\
tants» parmi les soignants, et identifie les facteurs \\
de risque de départs prématurés en comparant \\
les soignants qui ont quitté leur travail ou la pro- \\
fession infirmière avec les soignants qui sont res- \\
tés dans leur établissement un an après. \\
Méthode: Cette étude européenne trans- \\
nationale inclut 34587 infirmiers (au recueil \\
initial) travaillant dans 623 hôpitaux, maisons de \\
retraite, soins à domicile, et comprend deux \\
mesures avec un intervalle d'un an. Les échantillons \\
finaux pour l'analyse longitudinale incluent \\
I 40 I6 restants et 866 partants. Pour ce qui \\
concerne les partants, les soignants participant \\
ont du indiquer à quel point les facteurs per- \\
sonnels et professionnels avaient contribué à \\
leur décision de quitter leur institution.
\end{tabular}

\begin{tabular}{l} 
A B S I R A C I \\
\hline Longitudinal Analysis of Personal \\
and Work-Related Factors Associated \\
with Turnover among Nurses \\
Background:While many scholars have ack- \\
nowledged the relationship between personal \\
and work-related factors associated with job \\
search and actual turnover, there is a lack of \\
longitudinal designs that test this relationship \\
empirically. \\
Objectives:This longitudinal study examines \\
specific determinant factors that differentiate \\
between so-called 'stayers' and 'leavers' within \\
the nursing profession, and identifies risk fac- \\
tors for premature leaving by comparing nurses \\
who have left their job, or the nursing profes- \\
sion, with nurses who stay. \\
Methods: This cross-national exploratory \\
European survey included 34,587 nurses (base- \\
line measurement) working in 623 hospitals, nur- \\
sing homes, and home care institutions, and \\
comprised two measurements with a one-year \\
time interval.The final samples for the follow-up \\
measurement included I 4,0 I 6 stayers and 866 \\
leavers. As regards the leavers, the participating \\
nurses were asked to indicate to what extent per- \\
sonal and work-related factors had contributed to \\
the decision to leave their health care institution.
\end{tabular}




\section{RECHERCHE}

ANALYSE LONGITUDINALE DES FACTEURS PERSONNELS ET PROFESSIONNELS

ASSOCIÉS AU TURNOVER PARMI LES SOIGNANTS.

\begin{abstract}
RÉ SU MÉ (suite)
Résultats: Les scores d'intention de partir des soignants ont été plus élevés à la mesure initiale parmi ceux qui ont quitté l'organisation au court des 12 mois suivants que parmi ceux qui y sont restés $(24,0 \%$ versus $13,5 \%$. Une majorité de soignants $(86,8 \%)$ ont quitté leur profession volontairement. Les départs involontaires sont survenus plus souvent dans les pays de l'Europe de l'Est. Les soignants ont indiqués que les raisons de départ ont été: les conditions de travail (par exemple, les problèmes relationnels, les difficultés émotionnelles, la pression temporelle et les difficultés pour la qualité des soins, l'insatisfaction de l'utilisation de ses compétences et le manque d'autonomie, les difficultés d'horaires de travail, l'insatisfaction du salaire) ; les raisons familiales (soins aux personnes de la famille). Pour un nombre important de soignants, les raisons de santé et le désir de formation complémentaire ont contribué à la décision de partir.

Discussion: Les organisations de santé devraient porter attention à des mesures préventives afin de protéger le marché du travail potentiel. Des recommandations sont faites pour le développement des ressources humaines dans les organisations de santé et pour d'autres recherches empiriques, afin de mieux comprendre à quel point différentes politiques de santé dans les pays européens expliquent la variation dans l'issue concernant la stabilité des personnels.
\end{abstract}

Mots clés : turnover, infirmiers, soignants, restants versus partants, conditions de travail, Europe, étude longitudinale, design.

\section{INTRODUCTION}

La majorité des pays de l'Ouest de l'Europe doit faire face à un important manque d'infirmiers (Aiken, Clarke, Sloane, Sochalski, et Silber, 2002; Sjögren, Fochsen, Josephson et Lagerström, 2004) dû à un départ prématuré de la profession (voir Hasselhorn, Tackenberg et Muller, 2003, pour une étude approfondie sur le départ prématuré parmi les infirmiers en Europe). Il y a des évidences: une faible rétention des infirmiers est liée à une charge lourde et à un niveau élevé de burnout lui-même lié au travail ainsi qu’à l'insatisfaction (Aiken et al ; 2002). De plus, il y a un nombre insuffisant d'infirmiers entrant dans la profession et dans le marché du travail en raison de charges de travail, de satisfaction professionnelle et de compensations non attractives (Kirpal, 2004). Un nombre inadéquat

\begin{abstract}
Results: Nurses' intent-to-leave scores were higher in the baseline measurement among those who left the organization during the following 12 months, than among those who stayed (24.0\% versus $13.5 \%)$. A majority of nurses $(86.8 \%)$ left their profession voluntarily. Involuntary departure occurred more often in Eastern European countries. Nurses indicated that the reasons for leaving were: working conditions (e.g., relationship problems, emotional difficulties, time pressure and quality of care, dissatisfaction with use of one's competence and lack of autonomy, work schedule difficulties, and dissatisfaction with pay) ; family reasons (e.g., caring for relatives). For a considerable number of nurses, health reasons and the desire for continuing education added to their decision to leave.

Discussion: Health care organizations should pay attention to preventive measures in order to protect labor market potential. Recommendations are made for human resource development in health care organizations and for further empirical research to better understand to what extent different policy systems across the European countries explain variance in outcomes.
\end{abstract}

Key words : turnover, nurses, stayers versus leavers, working conditions, europe, longitudinal, design. d'infirmiers est une préoccupation publique du fait du turnover infirmier et du manque de soignants qui ont été associés à une diminution dans les standards de soins aux patients (Newman, Maylor, et Chansarkar, 2002) et à une augmentation de la pression sur ceux qui restent dans les soins (Borda et Norman, 1997).

Une manière efficace d'assurer un nombre suffisant de soignants est de retenir le personnel existant (EstrynBehar et al 2007a; Estryn-Behar et al 2007b; EstrynBehar et al 2008), de prévenir les départs prématurés du poste et de la profession et d'augmenter la rétention dans le secteur des soins de santé jusqu'à un âge raisonnable de départ en retraite (Armstrong-Stassen, 2005; Mion et al; 2006; Tourangeau et Cranley, 2006). Afin de mieux comprendre comment accroître le nombre de 


\section{ANALYSE LONGITUDINALE DES FACTEURS PERSONNELS ET PROFESSIONNELS ASSOCIÉS AU TURNOVER PARMI LES SOIGNANTS.}

soignants en poste, il est nécessaire de rechercher les facteurs qui différencient les soignants qui sont restés dans leur travail et les soignants qui ont quitté leur institution. Les études empiriques des facteurs qui influencent le turnover, fournissent une base pour des développements théoriques et d'autres recherches sur ce sujet dans une perspective de développement des ressources humaines (Peterson, 2004).

Le modèle qui visait pour l'ensemble de la recherche européenne PRESST-NEXT (Promouvoir en Europe Santé et Satisfaction des Soignants au Travail - Nurses' Early Exit Study), voir figure I (Hasselhorn,Tackenberg, et Muller, 2003), est basé sur l'affirmation que la décision individuelle de quitter le métier de soignant prématurément est fonction de(s) :

- la demande au travail (cercle I) et la vie privée (cercle 2); - modes d'exposition aux risques (rectangle 3, problème soudain ou problème chronique);

- conditions individuelles et des ressources (rectangle 4); - l'alternative possible (rectangle 5), par exemple : meilleur poste de travail ou salaire, opportunité de formation, pension d'invalidité, retraite prématurée, ou autre métier dans l'institution.

Ainsi, la décision de quitter la profession soignante semble résulter d'un processus avec de nombreuses causes sous-jacentes simultanées, c'est-à-dire, à la fois des facteurs qui poussent à rester et des facteurs qui attirent vers l'extérieur (Beehr, Glazer, Nielson, et Farmer, 2000). Les facteurs qui poussent vers la sortie sont les aspects du travail perçus de façon négative qui amènent l'employé à souhaiter quitter l'emploi qu'il occupe (par exemple conflits au travail ou problème de santé); les facteurs qui attirent sont des incitations à aller vers l'extérieur telles que les études universitaires ou les législations concernant la retraite.

La majorité des études précédentes sur les comportements professionnels infirmiers a insisté sur les facteurs organisationnels du turnover (Borda et Norman, 1997; Irvine et Evans, 1995) et moins sur le turnover lié au travail lui même (voir aussi Van der Heijden, Van Dam, et Hasselhorn, 2009). Des recherches plus empiriques sur le comportement professionnel sont nécessaires parce qu'elles permettent de comprendre le départ de la profession de façon plus globale, départs qui réduisent le nombre total de soignants actifs disponibles.

Les objectifs et les buts de cette étude ont été de : - identifier et classer les pays selon l'importance de l'intention d'abandonner la profession (IAP);

- déterminer l'importance de l'IAP initiale parmi les « restants » par rapport aux «partants»;

- comparer les caractéristiques des «partants» selon les pays;

- déterminer les principales raisons de départ;

- étudier les facteurs qui ont contribué à la décision de départ.

Figure 1.

Modèle utilisé pour la recherche européenne PRESST-NEXT

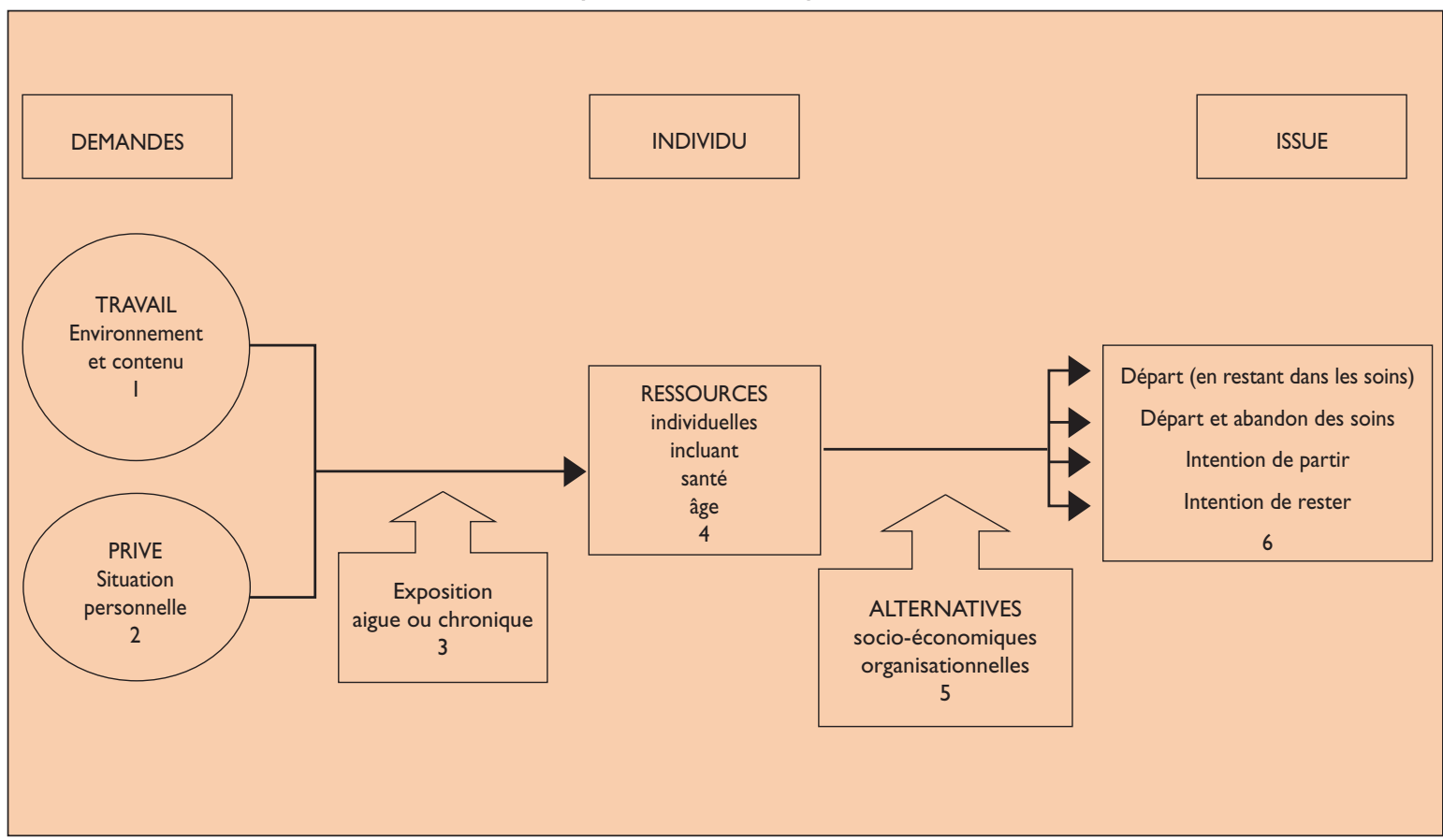




\section{MÉTHODE}

\section{Echantillon et procédure}

Cette étude inclut 623 établissements hospitaliers européens, maisons de retraite, et institutions de soins à domicile qui ont été sélectionnés de façon à représenter la distribution nationale (régionale) des infirmiers travaillant dans les différents types de structures parmi les pays européens participants. La répartition locale des soignants entre secteurs publics et privés a été aussi prise en compte ainsi qu'entre les institutions de soins aigus ou de moyens et longs séjours. De nombreuses institutions différentes ont été incluses pour prévenir l'effet de "niche" des données autant que possible.

Selon les pays, entre 5100 et I 3000 soignants paramédicaux de différentes qualifications (infirmiers diplômés sans poste de supervision, ou en position de supervision et aides-soignants) ont été approchés pour participer à notre étude. Pendant l'automne 2002, le questionnaire initial (QI) qui couvrait les questions se rapportant à la biographie professionnelle et à des conditions personnelles importantes (par exemple état de santé, obligation de donner des soins à des proches),et à l'environnement relationnel de travail, aux demandes professionnelles, à l'organisation du travail et aux projets professionnels des infirmiers, a été envoyé à tous les infirmiers dans les institutions participantes.

Dans la majorité des cas, les questionnaires ont été envoyés par les institutions elles-mêmes pour protéger les droits privés des employés. Les questionnaires complétés ont été retournés avec des enveloppes prépayées aux structures de recherche de chaque pays participant. Pendant les 12 mois suivants (un an de suivi longitudinal), tous les infirmiers qui ont quitté leur institution initiale ont reçu un questionnaire de «partants» (QP) immédiatement après leur départ, ce QP a été utilisé pour rechercher les raisons sousjacentes aux départs de ces infirmiers.

Les répondants qui sont restés dans leur institution, appelés «restants», ont été sollicités pour répondre au questionnaire de suivi 12 mois après $\left(\mathrm{QL}_{12}\right)$ le QI. Ce questionnaire a été utilisé pour rechercher les conséquences de la poursuite du même emploi et d'étudier les changements qui sont survenus. Les études $\mathrm{QI}$ et $\mathrm{QL}_{12}$ ont été des études extensives alors que celle du QP a été plus courte et avait un format plus spécifique; (voir Hasselhorn, Tackenberg et Müller, 2003 pour plus d'informations spécifiques sur le contenu de l'étude).

L'étude longitudinale NEXT a été approuvée par le Comité d'Ethique de l'université de Wuppertal en Allemagne (coordinateur de l'étude), et mise en place soigneusement en suivant les codes d'éthique et de conduite, en ce qui concerne l'anonymat de la restitution des résultats, au sein des huit pays participants (Belgique, Finlande, France, Allemagne, Italie, Pays-Bas, Pologne et Slovaquie).

Un code d'anonymat, choisi par les participants euxmêmes, a été utilisé afin de suivre les répondants et d'établir les liens entre les bases de données: pour les «restants», le questionnaire initial QI a été relié avec le questionnaire de suivi 12 mois après $\mathrm{QL}_{12}$, et pour les «partants», QI a été relié avec le questionnaire des «partants» QP.

\section{Mesures}

Pour avoir une meilleure compréhension des causes principales de départs prématurés, les «partants» participants ont dû indiquer leurs principales raisons de départ à partir de six raisons possibles:

- la formation continue dans les soins (précisant leur développement professionnel ultérieur);

- les conditions de travail;

- les raisons de santé;

- le salaire;

- le besoin de temps pour prendre soin de parents;

- autre raison privée.

Ensuite, les infirmiers participants ont dû indiquer l'importance des facteurs spécifiques personnels et professionnels qui ont contribué à la décision de quitter leur institution en soins de santé. Cette section de l'étude a été introduite par une question générale : «Pourquoi avez vous quitté votre lieu de travail? ॥ II était précisé de répondre à toutes les questions avec à l'esprit que plusieurs raisons peuvent amener à quitter un lieu de travail.

Les 63 facteurs proposés comprenaient une échelle de réponses allant de « pas du tout » à « dans une très large mesure », ils ont été agrégés en 15 catégories.

Dans les facteurs personnels, ont été inclus les problèmes travail/famille et les besoins familiaux, comprenant:

- la protection légale de la grossesse ou de la femme enceinte;

- la nécessité de prendre soin des enfants ou d'un proche;

- le besoin financier de travailler;

- le fait d'un changement de résidence du partenaire; et autre raison familiale.

Dans les facteurs liés au travail ont été pris en compte: - les problèmes relationnels avec l'employeur, le cadre supérieur, les collègues, les médecins, ou les subordonnés; les problèmes concernant le climat de travail; les problèmes concernant l'atmosphère générale et les questions de harcèlement; 


\section{ANALYSE LONGITUDINALE DES FACTEURS PERSONNELS ET PROFESSIONNELS ASSOCIÉS AU TURNOVER PARMI LES SOIGNANTS.}

- les difficultés émotionnelles comprennent le manque de soutien psychologique et des sollicitations émotionnelles trop importantes;

- la pression temporelle et la qualité des soins incluent le trop de responsabilité, le trop à faire, la crainte de commettre des erreurs, le fait d'avoir fait une erreur, le manque de temps auprès des patients, la réduction des moyens pour les soins aux patients, la pression temporelle, la qualité insatisfaisante du travail;

- le manque d'effectif a été également inclus comme facteur possible contribuant à la décision de partir;

- l'insatisfaction des possibilités d'utiliser sa compétence et d'exercer son autonomie comprend les variables comme: trop de responsabilité, peu de défi et d'enjeu professionnel, trop peu de latitude décisionnelle, travail trop simple, travail trop routinier et tâche non liée à son métier;

- l'insatisfaction des opportunités de développement offertes au travail;

- les difficultés d'horaires incluent des horaires insatisfaisants: trop de week-end, trop de travail de nuit, trop d'heures supplémentaires;

- l'insatisfaction du salaire comprend un salaire trop faible par rapport à ses besoins, au travail effectué, au salaire des infirmiers d'autres institutions, à d'autres professions ayant des niveaux d'études similaires et/ou à de mauvaises perspectives salariales.

L'intention d'abandonner la profession a été mesurée par un item: «A quelle fréquence, au cours de l'année précédente, avez vous pensé à abandonner les soins infirmiers? ॥ Les réponses étaient à cocher: jamais, quelque fois par an, quelque fois par mois, quelque fois par semaine ou chaque jour. Quelque fois par mois ou plus souvent ont été interprété comme un indicateur de fréquentes intentions d'abandonner ( $\mid 4,1 \%$ de l'échantillon total $Q \mid$ et I3,5\% de l'échantillon total $\mathrm{QL}_{12}$ ).

Les questionnaires du projet de recherche NEXT ont été établis par un groupe d'experts (médecins, infirmiers, psychologues et statisticiens) en se basant sur les recherches précédentes, sur des interviews avec des soignants dans trois pays, et sur trois études pilotes. La méthode de traduction inverse, avec vérification de la concordance de la traduction dans les différentes langues par une retraduction en Anglais, a été utilisée par tous les membres des pays, en utilisant la version anglaise de base (Hambleton, 1994). L'étude contient environ 260 questions (Hasselhorn et al., 2003) comprenant une biographie professionnelle, des questions concernant la vie privée, l'environnement social de travail, l'organisation du travail, l'exigence du travail et la pression temporelle, les ressources individuelles et les projets professionnels futurs.
Une description détaillée des échelles est donnée dans Estryn-Béhar et al. (2007), la majorité des questions ont été conservées pour $\mathrm{QI}_{12}$.

\section{Analyses}

Les analyses ont porté sur les principales raisons de départ et la contribution des facteurs personnels et professionnels à la décision de partir parmi :

- les soignants des trois catégories de pays;

- les soignants qui ont changé de lieux de travail comparés à ceux qui ont complètement quitté les soins.

Les analyses bi variées, utilisant le test de Chi-carré de Pearson, ont été conduites avec le logiciel SPSS I2.0, afin de déterminer l'influence des différents facteurs. Dans l'ensemble, les différentes questions ont eu peu de données manquantes; cependant quelques différences dans les tailles d'échantillons pour des analyses spécifiques peuvent être reliées à ces données manquantes.

\section{RÉSULTATS}

\section{Caractéristiques de l'échantillon}

Le taux de réponses pour les 61940 soignants approchés pour le Ql a été $55,87 \%$. Les caractéristiques de l'échantillon sont détaillées dans le tableau I (annexe). II y a eu 23517 « restants » et I526 « partants » pendant l'année de suivi longitudinal.

En utilisant les codes d'anonymat, il a été possible de relier deux mesures pour I4 016 "restants" (QI et $\left.\mathrm{QL}_{12}\right)$ et 866 «partants» (Ql et $\left.\mathrm{QP}\right)$.

L'échantillon final a été classé en trois catégories de pays selon l'importance de l'intention d'abandonner la profession (IAP) mesurée initialement par le QI. Le groupe I comprend les Pays Bas et la Belgique (faible IAP respectivement $8,8 \%$ et $9,8 \%$ ); le groupe 2 comprend la Finlande, la France, l'Allemagne et l'Italie (IAP prélevée respectivement: 14,2\%, I5,4\%, I8,4\%, 20\%); Le groupe 3 comprend les pays de l'Europe de l'Est, la Pologne et la Slovaquie (IAP moyenne II,0\% et II,7\%).

\section{Résultats démographiques préliminaires}

La moyenne d'âge des 23517 soignants est de 40,7 ans (écart-type ET=9,01). Leur ancienneté moyenne dans l'établissement était de 4,57 ans $(E T=0,85)$ (voir tableau 2 en annexe, pour les résultats spécifiques de chaque pays).

La majorité des « restants 》 étaient des femmes $(89,9 \%) ; 56,4 \%$ des « restants » vivaient avec un autre 
adulte et un enfant, $6,4 \%$ vivaient seuls avec un ou des enfants, $26,2 \%$ vivaient avec un autre adulte sans enfant, et II,0\% « des « restants » vivaient seuls.

Pour ce qui est des I 526 « partants ॥, la moyenne d'âge était de 38,95 ans $(E T=\mid I, 7)$, leur ancienneté moyenne était de 6,9l ans $(E T=7,0)$.

La majorité des «partants» étaient des femmes $(90,4 \%), 42,6 \%$ des « partants » vivaient avec un autre adulte et des enfants; $5,4 \%$ vivaient seuls avec un enfant, $36,5 \%$ vivaient avec un autre adulte sans enfant; $15,6 \%$ vivaient seuls.

\section{Intention d'abandonner parmi \\ les « restants » comparés \\ aux « partants "; résultat du questionnaire initial}

L'IAP était beaucoup plus élevée au QI parmi les soignants qui ont ensuite quitté leur organisation pendant les 12 mois de suivi, comparés avec l'IAP de ceux qui sont restés $(24,0 \%$ versus I $3,5 \%$, voir tableau I p.40). Les différences d'IAP entre les « partants ॥ par rapport aux « restants » étaient plus élevées dans le groupe I $(17,9 \%$ versus $7,9 \%=10 \%)$ et le groupe 2 (26,4\% versus $16,4 \%=10 \%)$ comparées avec la différence d'IAP entre les « partants » et les « restants » du groupe $3(14,3 \%$ versus $10,1 \%=4,2 \%)$.

\section{Caractéristiques des " restants ॥ selon les pays}

La vaste majorité des soignants $(86,8 \%)$ ont quitté leur profession volontairement (tableau 3 p. 42). Les départs involontaires (contrat non renouvelé, ou venant à sa fin ou licenciement) sont survenus plus souvent dans les pays de l'Europe de l'Est (15,8\% pour le groupe 3 versus 12,9\% pour le groupe I, et $12,7 \%$ pour le groupe $2 ; p<00 \mathrm{I}$ ).

Parmi les 3 groupes, les soignants qui eux-mêmes n'ont pas voulu renouveler leur contrat avaient travaillé pour cet employeur moins de deux ans. Les soignants qui ont démissionné avaient une ancienneté de 6 ans et avaient cherché à s'adapter aux circonstances, mais finalement avaient décidé de partir. Dans chaque groupe, les soignants qui ont pris du temps de disponibilité étaient restés environ 8 ans avec le même employeur et ont indiqué qu'ils avaient exploré s'ils pouvaient trouver une meilleure situation ailleurs et avaient maintenu l'opportunité de revenir s'ils ne réussissaient pas dans ce projet.

Un départ prématuré de la profession (retraite prématurée, nouveau métier ou formation hors des soins) a été identifié pour $26,7 \%$ de l'échantillon total. Les autres ( $73,4 \%$ des départs) ne sont pas considérés comme des départs prématurés des soins, mais un changement dans la carrière (retraite à l'âge normal, formation dans les soins, nouveau travail dans les soins ou recherche d'un nouveau travail dans les soins). Le départ prématuré est plus fréquent dans le groupe 3 (44,9\%), principalement du fait de retraites prématurées, comparés à $22,5 \%$ dans le groupe I et $23,8 \%$ dans le groupe $2(p<0,00 \mathrm{I})$.

\section{Principales raisons de départ de l'institution}

Lorsqu'ils ont été interrogés sur la raison principale de quitter leur institution de santé, la réponse la plus fréquemment rapportée dans les trois groupes a concerné les conditions de travail (groupe I : 36,8\%, groupe 2 :30, I\%, groupe 3 :29,3\%; tableau 4 p. 43 ). Parmi les soignants qui ont quitté leur institution sans quitter les soins, un plus grand nombre encore de soignants ont rapporté que les conditions de travail étaient la cause principale $(35,9 \%$ versus $23,1 \%)$ pour l'échantillon total et encore plus dans le groupe I (4I,4\% versus 19,6\%).

Les raisons privées (soins à la famille ou autres raisons privées) ont été citées par près d'un tiers de l'échantillon dans le groupe $2(30,6 \%)$, mais moins dans le groupe I $(24,2 \%)$ et dans le groupe $3(17,2 \%)$.

Le salaire a concerné un quart de l'échantillon dans les pays de l'Europe de l'Est, groupe 3, versus 6,5\% dans le groupe 2 et $0,7 \%$ dans le groupe I. Le salaire a été très important pour ceux qui ont complètement abandonné les soins dans le groupe 3 (31,7\% versus $21,9 \%)$ par rapport aux soignants qui sont restés dans les soins.

Les raisons de santé ont été plus prévalentes parmi les soignants du groupe 3 (I2,1\% versus $6,3 \%$ dans le groupe I et 7,4\% dans le groupe 2 ).

La formation continue, qui peut être interprétée comme une raison positive de quitter une institution, a été rapportée relativement plus souvent dans le groupe 2 ( $13,8 \%$ versus $10,2 \%$ dans le groupe I et $8,6 \%$ dans le groupe 3).

\section{Importance des facteurs contribuant à la décision de partir}

Les facteurs personnels et professionnels qui ont été pris en compte dans l'étude ont été classés selon leur importance (tableau 5 p. 44). La pression temporelle et des difficultés pour la qualité des soins ont été la catégorie de facteurs la plus fréquemment choisie, rapportée par plus de $70 \%$ des soignants qui 


\section{ANALYSE LONGITUDINALE DES FACTEURS PERSONNELS ET PROFESSIONNELS ASSOCIÉS AU TURNOVER PARMI LES SOIGNANTS.}

ont quitté parmi les groupes I et 2 . Elle a été au $3^{\text {ème }}$ rang dans le groupe 3 (plus de $60 \%$ des soignants ont considéré que ces items ont contribué très notablement). L'insatisfaction de l'utilisation de ses compétences et le manque d'autonomie ont eu le $2^{\text {ème }}$ rang dans les trois groupes de pays. L'insatisfaction du salaire a été la catégorie la plus fréquemment choisie dans le groupe 3; elle était au 3 ème rang pour le groupe 2 , au $5^{\text {ème }}$ rang pour le groupe I.

Les problèmes relationnels venant au $4^{\text {ème }}$ rang dans le groupe I et 2 , et au $6^{\text {ème }}$ dans le groupe 3 , ont concerné plus de la moitié des soignants qui ont quitté leur institution.

L'insuffisance d'effectifs a été au 5 ème rang pour l'ensemble, contribuant à la décision de partir pour $50 \%$ des soignants ( $5^{\text {ème }}$ rang dans le groupe $2,6^{\text {ème }}$ dans le groupe I et $7^{\text {ème }}$ dans le groupe 3 ).

L'insatisfaction des opportunités de développement a été la $6^{\text {ème }}$ catégorie de facteurs la plus fréquemment choisie, spécialement par les soignants du groupe 3 (4 ${ }^{\text {ème }}$ rang dans ce groupe).

Les difficultés d'horaires ont été au 7 ème rang pour l'ensemble, mais en rang 3 pour ceux qui ont quitté dans le groupe I $(57,9 \%)$; alors qu'il a été classé treizième dans l'ordre d'importance pour les soignants du groupe 3 (35,3\%).

Les difficultés émotionnelles ont été au 8 ème rang, rapportées par un peu plus de $50 \%$ des soignants dans le groupe 2 et 3 et par $49,6 \%$ du groupe I.

La charge physique a été la 9ème catégorie de facteurs en lien avec le départ prématuré.

Pour ce qui est des facteurs personnels, les problèmes entre famille et travail et les besoins de la famille ont été respectivement au I0ème et I lème rang. Ils ont été déclarés comme ayant contribué dans une large mesure à la décision des soignants de quitter par un peu plus du tiers des « partants » de chaque groupe, un peu plus parmi les « partants » du groupe I où il a eu le 7ème rang concernant $47,7 \%$ des soignants.

Les raisons de santé, au $14{ }^{\text {ème }}$ rang ont été rapportées comme ayant contribué de façon importante pour plus d'un tiers de l'échantillon dans le groupe 3 .

\section{DISCUSSION}

\section{Taux de réponse}

Plusieurs facteurs doivent être pris en compte pour le taux de réponses. Certaines institutions, dans certains cas de manière inattendue, ont décidé de ne pas participer au deuxième recueil. De plus, les soignants qui ont quitté la profession peuvent s'être distanciés de celle-ci, et n'ont plus été motivés pour participer à des mesures longitudinales. Enfin, les soignants qui sont restés et qui n'ont pas vu d'amélioration dans leurs conditions de travail peuvent avoir été moins disposés à participer à un second recueil.

\section{Réflexion concernant les résultats:}

Cette analyse longitudinale, utilisant les données de l'étude européenne PRESST-NEXT, a permis l'identification de facteurs de risques de départs prématurés de l'emploi de soignants. La force de cette étude est qu'elle analyse et compare l'intention d'abandonner la profession déclarée initialement et le départ effectif au cours de l'année suivante. Selon la théorie d'Ajzen (1991), concernant les comportements planifiés, nous avons débuté avec l'affirmation que l'intention de quitter la profession serait le plus proche prédicteur du turnover réel. Les recherches empiriques sur le turnover (par exemple Adams et Beehr, 1998, Griffeth, Hom, et Gaertner, 2000) corroborent que l'intention de turnover est un prédicteur très fort du turnover réel par rapport à d'autres variables (Blau et Lunz, 1998). L'intention d'abandonner la profession peut être considérée comme un indicateur de la tendance d'un individu à sortir d'une carrière spécifique (Hanisch et Hulin, 1990); elle permet de mener des analyses sur cette tendance alors que le turnover réel est un évènement moins fréquent et plus difficile à analyser. De plus, l'intention de changer de profession a été identifiée comme une variable importante pour sa propre santé (Blau et Lunz, 1998) car elle représente la composante décisionnelle consciente du changement (Rhodes et Doering, 1993).

Le pourcentage de soignants qui ont réellement quitté pendant la durée de l'étude était significativement plus élevé parmi les soignants qui pensaient à partir à la première mesure. De plus, la majorité des soignants ont quitté la profession volontairement, bien que les départs involontaires ont été relativement plus fréquents parmi les soignants de l'Europe de l'Est en comparaison avec les autres pays.

La première raison d'ensemble mise en avant par les soignants pour avoir quitté leur institution de soins, est les conditions de travail. Les raisons privées telles que les soins aux parents, et les conditions de salaire viennent ensuite.

Il y a plusieurs résultats principaux qui affectent la capacité d'assurer la disponibilité d'un nombre 
suffisant de soignants dans un avenir proche. Si les organisations de santé veulent se concentrer sur des mesures de prévention, il est important de porter attention aux thèmes suivants:

- l'équilibre famille/travail (Van der Heijden, Demerouti et Bakker, 2008);

- les problèmes de relations dans la sphère de travail;

- les difficultés émotionnelles liées à la situation de travail;

- la pression temporelle telle que perçue par les soignants et la qualité des soins qu'ils peuvent apporter dans ces circonstances;

- l'insatisfaction des soignants concernant la manière dont leurs compétences sont utilisées et l'autonomie qu'ils peuvent avoir;

- les difficultés d'horaires;

- l'insatisfaction du salaire.

De plus, étant donné les besoins actuels de porter attention à l'employabilité future et au potentiel de carrière (Van der Heijden et Van der Heijden, 2006), il est important de donner des opportunités de développements professionnels, à la fois par l'apprentissage au cours du travail et par des programmes plus formels de formation.

Les résultats de cette étude reconfirment les résultats de Tourangeau et Cranley (2006) et de Stone et al. (2003) qui ont trouvé, de la même manière, que les caractéristiques du travail sont importantes dans les intentions de départ. Dans beaucoup d'études, les conditions de travail sont le premier facteur pour la perte des potentialités du marché du travail dans le secteur des soins (Coomber et Barriball, 2007; De Lange, DeWitte et Notelaers, 2008; Griffeth, Hom, et Gaertner, 2000; Schaufeli, Taris, Le Blanc, Peeters, Bakker et De Jonge 200I).

En plus de ces résultats sans ambigüité, il y a des différences entre pays qui ont été observées dans le vaste contexte de l'étude NEXT (Hasselhorn, Tackenberg et Müller, 2003). Par exemple, les soignants du troisième groupe (Pologne et Slovaquie) ont rapporté un manque très important de matériel de manutention, un score élevé (la plus forte moyenne) d'invalidité liée au travail (due aux maux de dos), un sérieux manque d'opportunités professionnelles dans leur région, et ont évoqué plus fréquemment des contraintes économiques. II semble que, dans ces circonstances, les décisions dans ce marché du travail soient très différentes de celles par exemple des soignants hollandais, qui rapportent la plus grande disponibilité du matériel de manutention et la plus courte durée hebdomadaire de travail ainsi que la plus faible fréquence de déséquilibre efforts / récompense (Siegrist, 1996). Malgré tout, beaucoup de soignants hollandais considèrent le départ prématuré en lien avec un manque de challenge perçu, un déséquilibre travail/famille, des raisons de santé ou une mauvaise atmosphère d'équipe, pour mentionner certaines raisons majeures. Cependant les infirmières hollandaises n'ont pas indiqué l'insuffisance de salaire comme principale raison sérieuse de considérer leur départ (Hasselhorn, Tackenberg et Müller, 2003).

Dans le vaste contexte de l'étude NEXT, il y a des différences contextuelles intéressantes parmi les soignants des trois groupes de pays. En cas de départs prématurés, spécialement en Pologne, la retraite prématurée a été choisie, vraisemblablement en lien avec la possibilité relativement plus élevée d'accéder à cette option (possible depuis 2007). Les décisions de retraite dépendent de façon importante du marché du travail de chaque pays et du contexte politique, incluant les politiques de retraite. Davantage de recherches sont nécessaires pour investiguer en quoi la décision des soignants de quitter est influencée par le contexte politique (Hasselhorn, Tackenberg et Müller, 2003).

\section{Limitations et recommandations pour de nouvelles recherches}

Premièrement, du fait que toutes les variables ont été mesurées par des questionnaires auto administrés, il existe un biais de méthodes (Podsakoff, Mac Kenzie, Lee, et Podsakoff, 2003). Bien que les résultats n'indiquent pas d'intercorrélations excessives, la possibilité que ce biais soit survenu ne peut pas être éliminée. Pour augmenter la validité des résultats, des questionnaires auto administrés et des évaluations par les pairs ou les superviseurs devraient être combinés dans des recherches futures.

Deuxièmement, comme dans beaucoup d'études longitudinales, il y a une diminution de taille de l'échantillon. Bien que ceci ne soit pas surprenant, en raison de la longueur du questionnaire d'étude, on ne peut clairement déterminer comment la diminution des répondants entre le questionnaire initial (au temps I) et le questionnaire rempli après 12 mois (temps 2) a affecté ces résultats. En effet, qu'une partie des soignants non répondant peut avoir quitté la profession, il est cependant possible que ces résultats sous estiment de vraies relations. Des comparaisons plus approfondies entre les réponses au questionnaire initial des « restants » par rapport aux « partants » sont nécessaires pour tirer des conclusions plus sûres.

Une troisième limitation de l'étude concerne les lieux de l'enquête. Du fait que les données ont été collectées dans la profession soignante et seulement auprès des soignants, les résultats ne peuvent pas être généralisés à d'autres professions ou groupes professionnels. Cependant, la généralisation aux soignants dans d'autres organisations de soins semble 


\section{ANALYSE LONGITUDINALE DES FACTEURS PERSONNELS ET PROFESSIONNELS ASSOCIÉS AU TURNOVER PARMI LES SOIGNANTS.}

raisonnable du fait de la large taille de l'échantillon et de l'échantillonnage des répondants parmi différents types d'institutions de soins.

Des recherches empiriques ultérieures, utilisant des mesures multi-items validés (échelle) sont nécessaires pour étudier la stabilité ou les changements dans l'intention d'abandonner, en lien avec les changements de perception de la situation de travail, la santé et l'équilibre famille/travail.

De plus, des modèles d'équations structurelles peuvent être utilisés pour mieux comprendre les facteurs causaux dans cet ensemble de données et tester, plus formellement, les hypothèses concernant la contribution de certains facteurs de risques pour les départs prématurés.

Les approches cross-culturelles sont nécessaires afin de conclure plus sûrement en quoi les différents systèmes politiques entre les pays européens et globalement expliquent la variance des résultats. Il est important de comprendre que les conditions de travail, étant un déterminant majeur du bien-être au travail, peuvent être influencées positivement, si le management veut rechercher une approche intégrale qui éclaire beaucoup de pratiques importantes tout au long du cycle de la vie de travail.

En lien avec l'intérêt actuel pour les recherches de psychologie positive (Schaufeli et Bakker, 2004), des recherches visant à mettre en place des carrières équilibrées au plan de la santé, satisfaisantes et motivantes pourraient former la base des recherches ultérieures. La diversité de cette population de professionnels, l'augmentation de l'importance des autres aspects de la vie, tels que la qualité de vie avec la famille et les amis ou les différences dans les loisirs, le style de « coping » et la personnalité - pour en mentionner quelques uns - conduisent à penser à des thématiques d'études qui dépassent celle de l'âge de la retraite pris isolément, telles que les pics de carrière, les succès dans la carrière et les besoins de formation continue (Van der Heijden,2005).

\section{Implications pratiques}

Le management des organisations de santé devrait porter attention aux aspects interpersonnels dans le contexte de travail soignant, afin de prendre en compte les problèmes relationnels, et de stimuler les réseaux de supports sociaux. Les soignants peuvent contribuer à un environnement positif interpersonnel, en apportant à leurs collègues du support et en recherchant pour eux-mêmes du support social quand c'est nécessaire. La recherche a montré que, dans les situations de stress psychosocial, très prévalentes dans la profession soignante, les collègues et les superviseurs sont d'importantes sources de support (Beehr, Jex, Stacey et Murray, 2000 ; Frese, 1999).

Le bien-être des patients étant la valeur dominante parmi les infirmiers, il est important d'étudier cet aspect de leur travail afin de guider leur implication professionnelle et leur satisfaction au travail (Meyer,Allen et Smith, 1993). C'est seulement si les soignants sont attachés à leur profession et perçoivent leur institution de santé comme un lieu agréable pour travailler, qu'ils auront l'intention de rester dans la profession soignante. Il est difficile de comprendre pourquoi, dans une période de manque de soignants, la fidélisation des soignants et les potentiels de carrière sont si peu centres d'intérêt et de soutien (Fugate, Kinicki et Ashorth, 2004 ; Van der Heijden, 2002; Van der Heijden et Van der Heijden, 2006). Ce n'est pas seulement le bien-être des soignants durant leur carrière et les développements de carrière qui sont affectés, mais la qualité et la quantité de soins qui est fournie maintenant et qui sera fournie dans le futur qui sont en jeu.

A l'évidence, les institutions peuvent choisir parmi une grande variété de mesures pour compenser les conditions de travail négatives et pour soutenir les situations personnelles des soignants. Mieux porter attention à l'équilibre entre les préférences individuelles et les circonstances de la vie peut combattre les départs prématurés des soins.

\section{RÉFÉRENCES}

Adams, G. A., \& Beehr, T. A. (1998). Turnover and retirement: A comparison of their similarities and differences. Personnel Psychology, 51(3), 643-665.

Aiken, L. H., Clarke, S. P., Sloane, D. M., Sochalski, J., \& Silber, J. H. (2002). Hospital nurse staffing and patient mortality, nurse burnout, and job dissatisfaction. JAMA, 288(16), 1987-1993.

Ajzen, I. (1991). The theory of planned behavior. Organizational Behavior and Human Decision Processes, 50(2), 179-211.

Armstrong-Stassen, M. (2005). Human resource management strategies and the retention of older RNs. Nursing Leadership, 18(1), 50-64. 
Beehr, T. A., Jex, S. M., Stacey, B. A., \& Murray, M. A. (2000). Work stressors and coworker support as predictors of individual strain and job performance. Journal of Organizational Behavior, 21(4), 391-405.

Beehr, T. A., Glazer, S., Nielson, N. L., \& Farmer, S. J. (2000). Work and nonwork predictors of employees' retirement ages. Journal of Vocational Behavior, 57(2), 206-225.

Blau, G. J., \& Lunz, M. E. (1998). Testing the incremental effect of professional commitment on intent to leave one's profession beyond the effects of external, personal, and work-related variables. Journal of Vocational Behavior, 52(2), 260-269.

Borda, R. G., \& Norman, I. J. (1997). Factors influencing turnover and absence of nurses: A research overview. International Journal of Nursing Studies, 34(6), 385-394.

Boyle, D. K., Bott, M. J., Hansen, H. E., Woods, C. Q., \& Taunton, R. L. (1999). Managers' leadership and critical care nurses' intent to stay. American Journal of Critical Care, 8(6), 361-371.

Bretz, R. D., Boudreau, J. W., \& Judge, T. A. (1994). Job search behavior of employed managers. Personnel Psychology, 47(2), 275-301.

Coomber, B., \& Barriball, K. L. (2007). Impact of job satisfaction components on intent to leave and turnover for hospital-based nurses: A review of the research literature. International Journal of Nursing Studies, 44(2), 297-314.

De Lange, A. H., De Witte, H., \& Notelaers, G. (2008). Should I stay or should I go? Examining longitudinal relations among job resources and work engagement for stayers versus movers. Work \& Stress, 22(3), 201-223.

European Commission. (1999). Employment observatory trends (No. 33 - Winter). Berlin: Author.

Estryn-Béhar, M., Le Nezet, O., Van der Heijden, B. I. J. M., Ogińska, H., Camerino, D., Conway, P. M., et al. (2007). Inadequate teamwork and burnout as predictors of intent to leave nursing according to seniority. Stability of associations in a one-year interval in the European NEXT Study. Ergonomia. An International Journal of Ergonomics and Human Factors, 29(3/4), 225-233.

Estryn-Béhar, M., Van der Heijden, B. I. J. M., Camerino, D., Fry, C., Le Nezet, O., Conway, P. M., et al. (2008). Violence risks in nursing. Results from the European NEXT Study. Occupational Medicine, 58(2), 107-114.

Estryn-Béhar, M., Van der Heijden, B. I. J. M., Ogifska, H., Camerino, D., Le Nezet, O., Conway, P. M., et al. (2007). The impact of social work environment, teamwork characteristics, burnout, and personal factors upon intent to leave among European nurses. Medical Care, 45(10), 939-950.

Frese, M. (1999). Social support as a moderator of the relationship between work stressors and psychological dysfunctioning: A longitudinal study with objective measures. Journal of Occupational Health Psychology, 4(3), 179-192.

Fugate, M., Kinicki, A. J., \& Ashforth, B. E. (2004). Employability: A psycho-social construct, its dimensions, and applications. Journal of Vocational Behavior, 65(1), 14-38.

Griffeth, R. W., Hom, P. W., \& Gaertner, S. (2000). A meta-analysis of antecedents and correlates of employee turnover: Update, moderator tests, and research implications for the next millennium. Journal of Management, 26(3), 463-488.

Hanisch, K. A., \& Hulin, C. L. (1990). Job attitudes and organizational withdrawal: An examination of retirement and other voluntary withdrawal behaviors. Journal of Vocational Behavior, 37(1), 60-78.

Hambleton, R.K. (1994). Guidelines for adapting educational and psychological tests: A progress report. European Journal of Psychological Assessment (Bulletin of the International Test Commission), 10, 229-244.

Hasselhorn, H., Tackenberg, P., \& Müller, B. (Eds.). (2003). Working conditions and intent to leave the profession among nurses staff in Europe. Report no. 2003: 7. A research project initiated by SALTSA (Joint Programme for Working Life Research in Europe) and funded by the European Commission (QLK6-CT2001-00475).

Hom, P. W., Caranikas-Walker, F., Prussia, G. E., \& Griffeth, R. W. (1992). A meta-analytical structural equations analysis of a model of employee turnover. Journal of Applied Psychology, 77(6), 890-909.

Kirpal, S. (2004). Work identities of nurses. Between caring and efficiency demands. Career Development International, 9(3), 274-304. 


\section{ANALYSE LONGITUDINALE DES FACTEURS PERSONNELS ET PROFESSIONNELS ASSOCIÉS AU TURNOVER PARMI LES SOIGNANTS.}

Irvine, D. M., \& Evans, M. G. (1995). Job satisfaction and turnover among nurses: Integrating research findings across studies. Nursing Research, 44(4), 246-253.

Mano-Negrin, R., \& Tzafrir, S. S. (2004). Job search modes and turnover. Career Development International, 9(5), 442-458.

Meyer, J. P., Allen, N. J., \& Smith, C. A. (1993). Commitment to organizations and occupations: Extension and test of a three-component conceptualization. Journal of Applied Psychology, 78(4), 538-551.

Mion, L. C., Hazel, C., Cap, M., Fusilero, J., Podmore, M. L., \& Szweda, C. (2006). Retaining and recruiting mature experienced nurses: A multicomponent organizational strategy. Journal of Nursing Administration, 36(3), 148-154.

Newman, K., Maylor, U., \& Chansarkar, B. (2002). The nurse satisfaction, service quality and nurse retention chain: Implications for management of recruitment and retention. Journal of Management in Medicine, 16(4), 271-291.

North, N., Rasmussen, E., Hughes, F., Finlayson, M., Ashton, T., Campbell, T., et al. (2005). Turnover amongst nurses in New Zealand's district health boards: A national survey of nursing turnover and turnover costs. New Zealand Journal of Employment Relations, 30(1), 49-62.

Peterson, S. L. (2004). Toward a theoretical model of employee turnover: A human resource development perspective. Human Resource Development Review, 3(3), 209-227.

Podsakoff, P. M., MacKenzie, S. B., Lee, J. Y., \& Podsakoff, N. P. (2003). Common method biases in behavioral research: A critical review of the literature and recommended remedies. The Journal of Applied Psychology, 88(5), 879 -903 .

Remery, C., Henkens, K., Schippers, J., \& Ekamper, P. (2003). Managing an aging workforce and a tight labor market: Views held by Dutch employers. Population Research and Policy Review, 22(1), 21-40.

Rhodes, S. R., \& Doering, M. (1993). Intention to change careers: Determinants and process. Career Development Quarterly, 42(2), 76-92.

Schaufeli, W. B., \& Bakker, A. B. (2004). Job demands, job resources, and their relationship with burnout and engagement: A multi-sample study. Journal of Organizational Behavior, 25(3), 293-315.

Schaufeli, W. B., Taris, T. W., Le Blanc, P., Peeters, M., Bakker, A. B., \& De Jonge, J. (2001). Maakt arbeid gezond? Op zoek naar de bevolgen werknemer [Does work make happy? In search of the engaged worker]. De Psycholoog, 36, 422-428.

Siegrist, J. (1996). Adverse health effects of higheffort/low-reward conditions. Journal of Occupational Health Psychology, 1(1), 27-41.

Sjögren, K., Fochsen, G., Josephson, M., \& Lagerström, M. (2004). Reasons for leaving nursing care and improvements needed for considering a return: A study among Swedish nursing personnel. International Journal of Nursing Studies, 42(7), 751-758.

Stone, P. W., Tourangeau, A. E., Duffield, C. M., Hughes, F., Jones, C. B., O'Brien-Pallas, L., et al. (2003). Evidence of nurse working conditions: A global perspective. Policy, Politics, \& Nursing Practice, 4(2), 120-130.

Tourangeau, A. E., \& Cranley, L. A. (2006). Nurse intention to remain employed: understanding and strengthening determinants. Journal of Advanced Nursing, 55(4), 497-509.

Van Dalen, E., Gründemann, R., \& De Vries, S. (2003). Onderweg naar morgen: Ontwikkelingen en hun betekenis voor toekomstig HRM [Along the way to tomorrow: Developments and their meaning for future HRM]. Tijdschrift voor HRM, 3, 63-92.

Van der Heijden, B. I. J. M. (2002). Prerequisites to guarantee life-long employability. Personnel Review, 31(1), 44-61.

Van der Heijden, B. I. J. M. (2005). "No one has ever promised you a rose garden" On shared responsibility and employability enhancing strategies throughout careers. Inaugural address delivered in abridged form on the occasion of the public acceptance of the professorship in Strategic HRM. Heerlen: Open University of the Netherlands. Assen: Van Gorcum. 
Van der Heijden, B.I.J.M., Demerouti, E., \& Bakker, A.B. (2008). Work-home interference among nurses: reciprocal relationships with job demands and health. Journal of Advanced Nursing, 62(5), 572-584.
Van der Heijden, B.I.J.M., Van Dam, K., \& Hasselhorn, H.M. (2009). Intention to leave nursing: The importance of interpersonal work context, work-home interference, and job satisfaction beyond the effect of occupational commitment. Career Development International, 14(7), 616-635.

\section{Remerciements}

L'étude Next est financée par la commission européenne dans le cadre du 5ème programme projet ID QLK-6-CT-200I-00475 et est coordonné au plan académique par le Dr Hans Martin Hasselhorn de l'Université de Wuppertal en Allemagne. En France, l'étude Next a été également soutenue par le Conseil Régional d'lle de France et le Conseil Régional de Rhône-Alpes. Les auteurs remercient Marie-André Vigil-Ripoche pour sa relecture attentive et ses conseils. 
ANALYSE LONGITUDINALE DES FACTEURS PERSONNELS ET PROFESSIONNELS ASSOCIÉS AU TURNOVER PARMI LES SOIGNANTS.

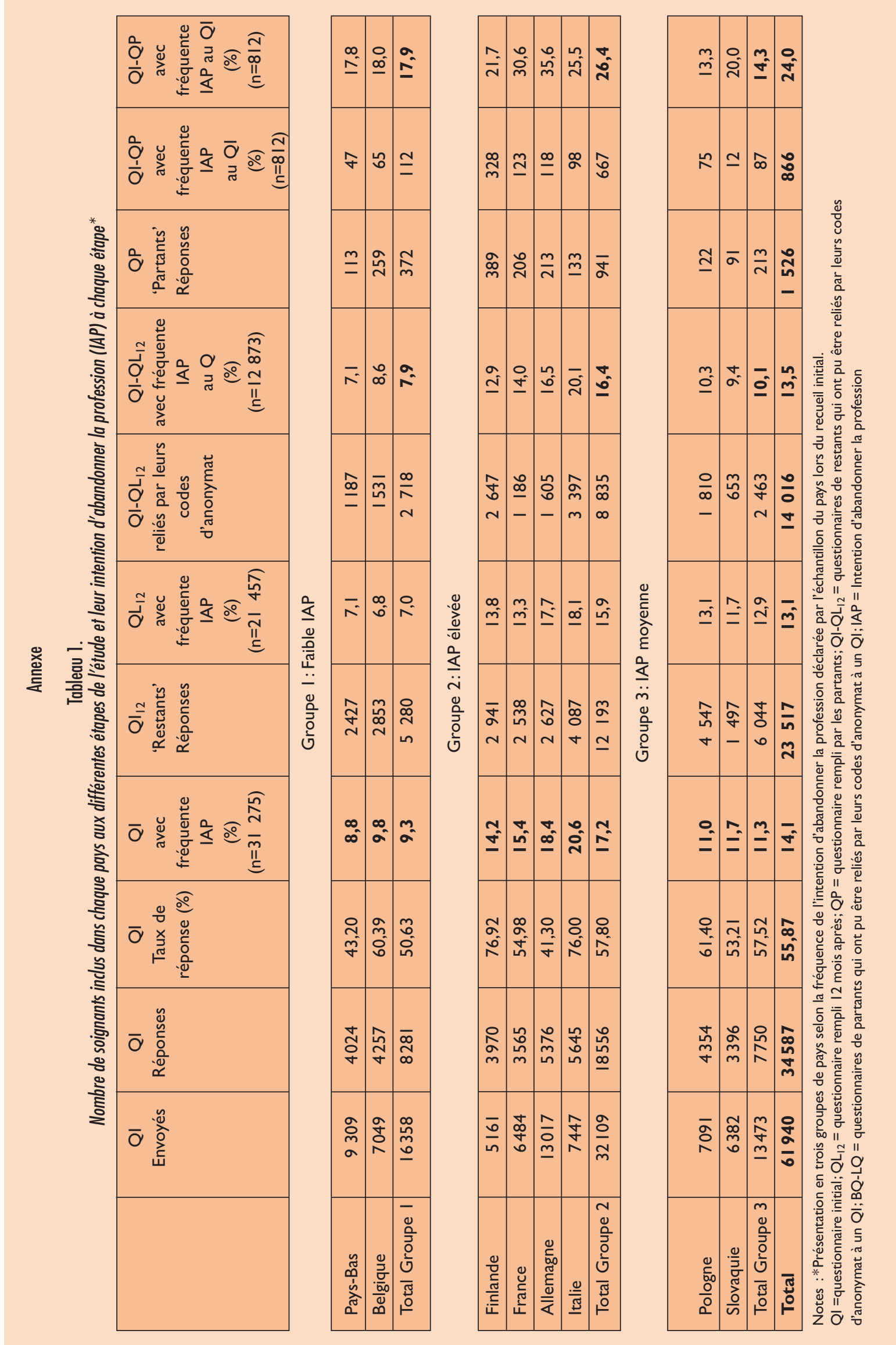




\begin{tabular}{|c|c|c|c|c|c|c|c|c|c|c|c|c|c|c|c|}
\hline \multirow{4}{*}{\multicolumn{2}{|c|}{ 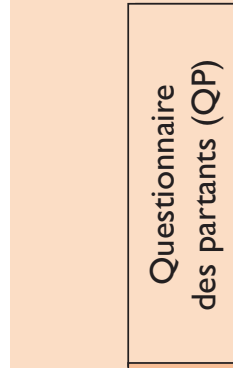 }} & $I \subsetneq$ & \multirow{25}{*}{$\begin{array}{l}\frac{0}{5} \\
\frac{0}{0} \\
\frac{0}{0} \\
\frac{10}{0} \\
\frac{0}{0} \\
\frac{0}{0} \\
\frac{0}{0}\end{array}$} & 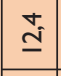 & 三 & \multirow{25}{*}{ 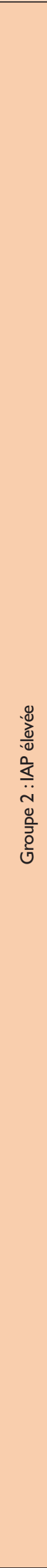 } & $\stackrel{\sim}{m}$ & \begin{tabular}{|l}
0 \\
$\underline{0}$
\end{tabular} & $\stackrel{=}{=}$ & $\frac{0}{i}$ & \multirow{25}{*}{ 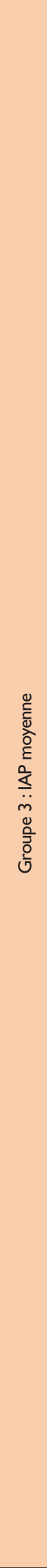 } & $\stackrel{\sim}{N}$ & : & $\stackrel{\circ}{\circ}$ & \multirow{25}{*}{ 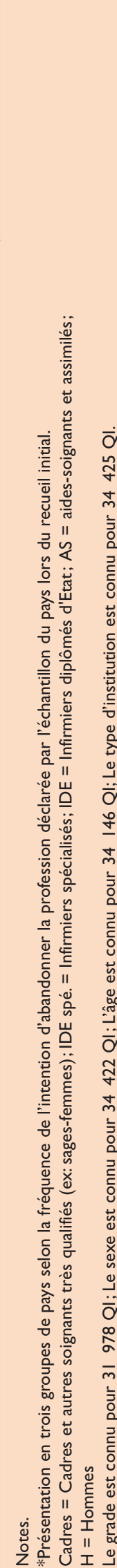 } \\
\hline & & $\stackrel{8}{8}$ & & $\begin{array}{l}\sigma \\
\text { 。. }\end{array}$ & స్ & & $\begin{array}{l}\stackrel{2}{0} \\
\stackrel{=}{=}\end{array}$ & $\stackrel{\underline{\tilde{N}}}{=}$ & 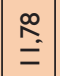 & $\bar{\sigma}$ & & \% & $\stackrel{\overline{\underline{n}}}{=}$ & $\stackrel{=}{=}$ & \\
\hline & & 哭啇 & & & \begin{tabular}{|c}
$\stackrel{m}{m}$ \\
$\stackrel{m}{m}$
\end{tabular} & & 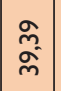 & 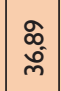 & 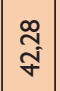 & $\begin{array}{l}0 \\
\stackrel{0}{0} \\
\infty_{m}^{\infty}\end{array}$ & & $\underset{\substack{\infty \\
\stackrel{\sigma}{\sigma}}}{ }$ & $\begin{array}{c}\infty \\
\stackrel{f}{f} \\
\mathcal{f}\end{array}$ & 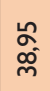 & \\
\hline & & 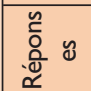 & & $\stackrel{m}{=}$ & 今े & & 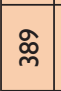 & $\frac{m}{\sim}$ & $\stackrel{\sim}{\circ}$ & $\underline{\underline{m}}$ & & $\Xi$ & $\bar{\sigma}$ & స్రి & \\
\hline \multirow{21}{*}{ 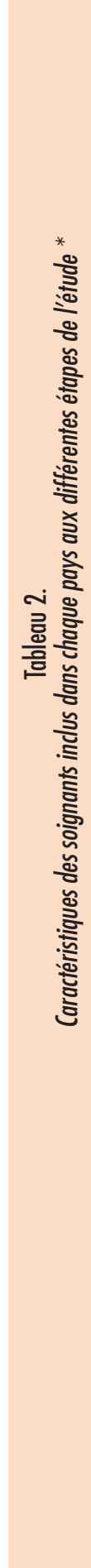 } & \multirow{21}{*}{ 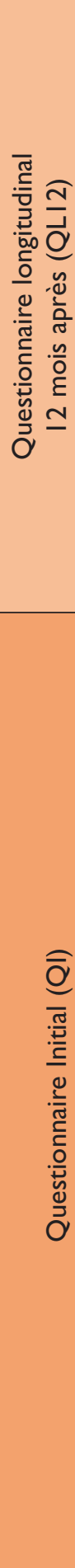 } & I & & to & $\stackrel{m}{\infty^{*}}$ & & $\bar{n}$ & $\stackrel{ }{\simeq}$ & $\underline{\underline{f}}$ & 望 & & $\underline{\underline{n}}$ & $\stackrel{ }{-}$ & $\overline{\bar{s}}$ & \\
\hline & & $\stackrel{8}{4} \bar{E}$ & & $\underset{\infty}{\infty}$ & $\stackrel{2}{\circ}$ & & 商 & & 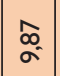 & $\underset{\sim}{\stackrel{N}{N}}$ & & 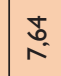 & \begin{tabular}{|l|l}
0 \\
$\infty$ \\
$\infty$
\end{tabular} & $\bar{\sigma}$ & \\
\hline & & 总高 & & $\begin{array}{l}\qquad 0 \\
\stackrel{0}{\circ} \\
\end{array}$ & 胥 & & $\begin{array}{l}\text { m. } \\
\text { భे }\end{array}$ & & 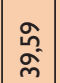 & $\begin{array}{l}\text { hn } \\
\text { à }\end{array}$ & & वृ & \begin{tabular}{|c|} 
\\
$m$ \\
$\dot{q}$ \\
$\dot{q}$
\end{tabular} & $\begin{array}{l}\hat{\hat{\alpha}} \\
\hat{\sigma}\end{array}$ & \\
\hline & & $<\subsetneq$ & & $\stackrel{\sigma}{\underline{m}}$ & $\hat{\sigma}$ & & $\hat{\bar{m}}$ & O̊ & $\mid \begin{array}{l}\infty \\
\stackrel{0}{0}\end{array}$ & $\tilde{m}$ & & $\stackrel{m}{0}$ & $\stackrel{m}{=}$ & $\stackrel{\circ}{I}$ & \\
\hline & & $\stackrel{\mathrm{Q}}{\mathrm{g}}$ & & $\widehat{\hat{D}^{\circ}}$ & 굼 & & $\stackrel{\sim}{\tilde{\sim}}$ & $\hat{\circ}$ & 点 & $\frac{0}{\infty}$ & & $\tilde{\infty}$ & 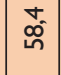 & مे & \\
\hline & & 㟔 。 & & $\stackrel{ \pm}{ \pm}$ & $\underline{\hat{0}}$ & & $\dot{\bar{\lambda}}$ & 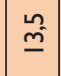 & $\hat{\mathrm{i}}$ & $\stackrel{i}{i}$ & & $\stackrel{0}{0}$ & in & $\underset{\mathcal{I}}{\underline{\Psi}}$ & \\
\hline & & 党 & & $\stackrel{\circ}{m}$ & $\underset{ \pm}{ \pm}$ & & $\stackrel{\forall}{=}$ & $\hat{\underline{a}}$ & $\bar{i}$ & $\stackrel{\stackrel{\sim}{\underline{m}}}{2}$ & & Oे & $\overline{\tilde{I}^{*}}$ & $\stackrel{m}{\underline{m}}$ & \\
\hline & & 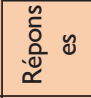 & & $\begin{array}{r}\hat{\mathfrak{J}} \\
\mathrm{N}\end{array}$ & $\stackrel{\substack{\infty \\
\sim}}{\sim}$ & & $\begin{array}{l}\bar{\Xi} \\
\sim\end{array}$ & $\mid \begin{array}{c}\infty \\
\stackrel{\infty}{\hat{~}} \\
\sim\end{array}$ & $\begin{array}{l}\hat{\mathbf{b}} \\
\text { in }\end{array}$ & $\begin{array}{l}\hat{\text { o }} \\
\text { वे } \\
\end{array}$ & & \begin{tabular}{c} 
f \\
\multirow{\sigma}{\sigma}{} \\
+
\end{tabular} & 产 & \begin{tabular}{|l}
$\hat{\Lambda}$ \\
$\tilde{\lambda}$
\end{tabular} & \\
\hline & & 妾 & & & & & $\begin{array}{l}0 \\
\underline{0}\end{array}$ & & & & & 全 & & $\hat{m}$ & \\
\hline & & 客 。 & & $\stackrel{\underline{\underline{0}}}{\underline{0}}$ & $\begin{array}{l}\infty \\
\substack{\infty \\
m}\end{array}$ & & & 음 & $\stackrel{2}{=}$ & $\stackrel{t}{=}$ & & $\bar{\kappa}$ & $\vec{\sim}$ & $\stackrel{\cong}{=}$ & \\
\hline & & $\frac{\alpha}{\Sigma} \subsetneq$ & & 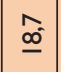 & $\bar{n}$ & & $\begin{array}{l}0 \\
\underline{0}\end{array}$ & $\underline{\underline{m}} \underline{\underline{m}}$ & $\begin{array}{l}0 \\
\dot{I}\end{array}$ & $\stackrel{n}{m}$ & & $\mathcal{F}$ & $\begin{array}{l}\text { O } \\
\text { 王 }\end{array}$ & $\bar{E}$ & \\
\hline & & $\begin{array}{l}\overline{\hat{o}} \\
\text { ㅇㅇ }\end{array}$ & & $\stackrel{\infty}{\widetilde{\sigma}}$ & $\overline{\mathrm{f}}$ & & $\begin{array}{l}0 \\
\infty_{0}^{0}\end{array}$ & $\begin{array}{l}\infty \\
\stackrel{\infty}{\uparrow}\end{array}$ & 志 & ल्ळ & & $\stackrel{\circ}{i}$ & $\begin{array}{c}\hat{\infty} \\
\infty\end{array}$ & 足 & \\
\hline & & I & & $\alpha^{2}$ & $\stackrel{\omega^{\circ}}{\infty}$ & & in & \begin{tabular}{|l|}
$\underline{n}$ \\
$\underline{\underline{\rho}}$
\end{tabular} & $\stackrel{\cong}{=}$ & ๗े & & $\stackrel{-}{-}$ & $\stackrel{m}{i}$ & $\hat{\underline{O}}$ & \\
\hline & & 造言 & & 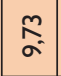 & $\stackrel{ \pm}{\Delta}$ & & $\widehat{\infty}_{0}$ & $\begin{array}{l}\hat{\tilde{z}} \\
\sigma\end{array}$ & స్్․ & న్ & & $\stackrel{m}{\stackrel{m}{N}}$ & \begin{tabular}{|c|c}
$\infty$ \\
$\infty$ \\
$\infty$
\end{tabular} & $\frac{\pi}{\sigma}$ & \\
\hline & & 桇产 & & 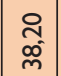 & 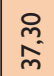 & & $\begin{array}{l}\overline{\mathcal{f}} \\
\bar{f}\end{array}$ & 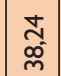 & $\frac{a}{9}$ & $\frac{m}{\infty}$ & & $\underset{\substack{\mathbb{N} \\
\text { No }}}{\mathbb{N}}$ & $\left|\begin{array}{l|}\infty \\
\infty \\
\infty \\
\infty \\
\infty\end{array}\right|$ & \begin{tabular}{|l}
$\stackrel{\infty}{\infty}$ \\
$\infty$ \\
$\stackrel{\infty}{\infty}$
\end{tabular} & \\
\hline & & భ o & & $\stackrel{\infty}{\stackrel{\infty}{\circ}}$ & $\stackrel{\circ}{=}$ & & $\stackrel{*}{*}$ & $\approx$ & $\begin{array}{l} \pm \\
0\end{array}$ & $\therefore$ & & $\stackrel{\Delta}{0}$ & $\stackrel{q}{q}$ & $\stackrel{m}{=}$ & \\
\hline & & $\stackrel{u}{\underline{\varrho}}$ & & 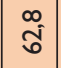 & 柋 & & $\bar{i}$ & $\mid \begin{array}{l}0 \\
\infty \\
\rho^{\circ}\end{array}$ & \begin{tabular}{c}
$\vdots$ \\
\multirow{\infty}{*}{} \\
$\infty$
\end{tabular} & $\underset{\infty}{\mathfrak{\infty}}$ & & 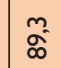 & 吕 & 苞 & \\
\hline & & 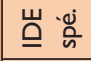 & & ì & ôे & & $\begin{array}{l}\dot{L} \\
\dot{\xi} \\
\dot{f}\end{array}$ & $\tilde{I}$ & $\because$ & $\infty^{0}$ & & : & $\hat{0}$ & \begin{tabular}{|l}
$\stackrel{0}{\underline{m}}$ \\
\end{tabular} & \\
\hline & & $\frac{\mathrm{g}}{\mathrm{c}} \mathrm{\sigma}$ 。 & & : & $\stackrel{\infty}{\sigma}$ & & $\stackrel{⿱ 乛}{+}$ & $\stackrel{\text { : }}{\circ}$ & 气̊ & $\approx \alpha$ & & $\stackrel{ \pm}{\subseteq}$ & $\overline{\underline{I}}$ & $\hat{\sigma}$ & \\
\hline & & 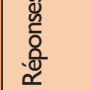 & & \begin{tabular}{l}
\multirow{J}{\vdots}{} \\
$\dot{\sigma}$
\end{tabular} & 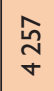 & & $\begin{array}{l}\text { 尺 } \\
\text { m }\end{array}$ & 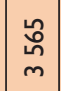 & 总 & $\begin{array}{l}\text { 号 } \\
\text { in }\end{array}$ & & 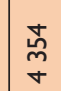 & $\begin{array}{c}\stackrel{\circ}{\rho} \\
\text { m }\end{array}$ & $\mid \begin{array}{l}\widehat{\infty} \\
\text { 岱 }\end{array}$ & \\
\hline & & & & 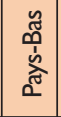 & 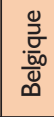 & & 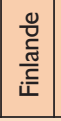 & 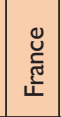 & 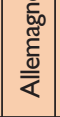 & $\frac{\underline{\underline{\underline{\omega}}}}{\underline{\underline{\underline{I}}}}$ & & $\begin{array}{l}\frac{0}{\tilde{\sigma}_{0}} \\
\frac{0}{0}\end{array}$ & 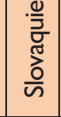 & 苞 & \\
\hline
\end{tabular}




\begin{tabular}{|c|c|c|c|c|c|c|c|c|c|c|c|c|c|c|}
\hline \multirow{8}{*}{ 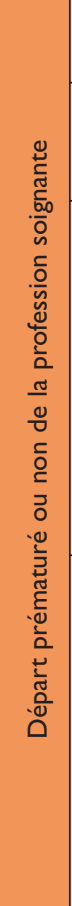 } & & a & & & 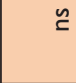 & & & & & $\begin{array}{l}\bar{\delta} \\
v^{\prime}\end{array}$ & & & $\begin{array}{l}\overline{8} \\
v^{\prime}\end{array}$ & $\bar{o}$ \\
\hline & & $=$ & 므 & İ & $\bar{m}$ & న్ & $\underline{\underline{\omega}}$ & 岕 & $\stackrel{\nabla}{=}$ & $\stackrel{\infty}{\gtrless}$ & $\stackrel{\infty}{=}$ & $\infty$ & $\stackrel{\sim}{\sim}$ & 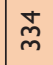 \\
\hline & \multirow{3}{*}{ 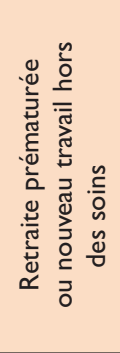 } & 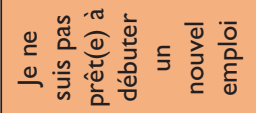 & @̊ & సे & $\begin{array}{l}\stackrel{\circ}{\circ} \\
\stackrel{+}{*}\end{array}$ & $\begin{array}{l}\stackrel{\circ}{0} \\
\underline{0} \\
\stackrel{f}{0}\end{array}$ & $\stackrel{\stackrel{\circ}{\circ}}{\underset{f}{*}}$ & $\begin{array}{l}\stackrel{\circ}{\wedge} \\
\hat{i}\end{array}$ & $\begin{array}{l}\stackrel{\circ}{\circ} \\
\vdots \\
0\end{array}$ & 苍 & ڤัํำ & 号 & ㅇํㄹ & 今̊ \\
\hline & & 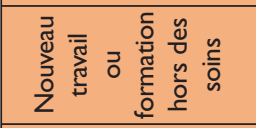 & $\stackrel{\stackrel{\circ}{2}}{\stackrel{m}{m}}$ & 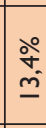 & $\stackrel{\stackrel{\circ}{\stackrel{\circ}{m}}}{\stackrel{0}{-}}$ & 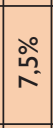 & 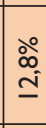 & $\begin{array}{l}\stackrel{0}{0} \\
0 \\
0 \\
0\end{array}$ & 官 & 总 & @ & 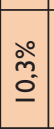 & $\begin{array}{l}\stackrel{\circ}{\circ} \\
\infty\end{array}$ & $\stackrel{\circ}{=}$ \\
\hline & & 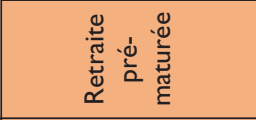 & 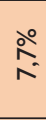 & 总 & $\frac{\circ}{\omega^{\circ}}$ & $\begin{array}{l}\stackrel{0}{0} \\
\vdots \\
0\end{array}$ & $\begin{array}{l}\stackrel{\circ}{+} \\
\dot{ \pm}\end{array}$ & $\begin{array}{l}\stackrel{0}{\circ} \\
\stackrel{+}{*}\end{array}$ & $\begin{array}{c}\text { ⿳ें日 } \\
\text { ஸे }\end{array}$ & 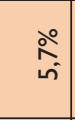 & ڤે & 品 & $\begin{array}{l}\stackrel{\circ}{\circ} \\
\dot{m} \\
\dot{m}\end{array}$ & $\begin{array}{l}\text { O̊ } \\
\text { O̊ }\end{array}$ \\
\hline & \multirow{3}{*}{ 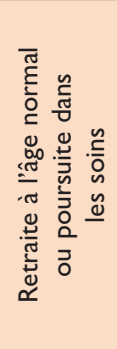 } & 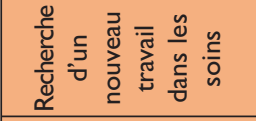 & $\stackrel{\stackrel{\circ}{\circ}}{{ }_{\leftarrow}^{\circ}}$ & ڤ̊ำ & ฏें & $\begin{array}{l}\stackrel{\circ}{\circ} \\
\stackrel{-}{\circ}\end{array}$ & $\stackrel{\circ}{-}$ & $\stackrel{\stackrel{\circ}{\stackrel{\circ}{*}}}{=}$ & $\begin{array}{l}\stackrel{\circ}{\circ} \\
\text { : }\end{array}$ & 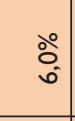 & $\frac{\circ}{\text { in }}$ & 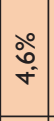 & $\stackrel{\circ}{\circ}$ & 今े \\
\hline & & 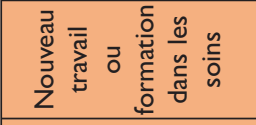 & $\stackrel{\circ}{\stackrel{2}{2}}$ & 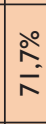 & $\stackrel{\stackrel{\circ}{+}}{\stackrel{\circ}{R}}$ & ì & 学 & ò. & 产 & $\begin{array}{l}\stackrel{\circ}{\circ} \\
\stackrel{\circ}{\circ}\end{array}$ & 息 & 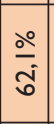 & 弚 & i̊n. \\
\hline & & 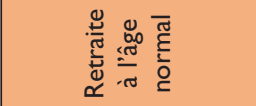 & & $\begin{array}{l}\stackrel{\circ}{\circ} \\
\text { ○े }\end{array}$ & ڤ̊ & $\stackrel{\stackrel{\circ}{=}}{=}$ & $\frac{\stackrel{\circ}{\sim}}{\stackrel{\sim}{*}}$ & 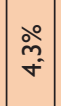 & $\begin{array}{c}\stackrel{\circ}{\circ} \\
\infty \\
\infty\end{array}$ & $\stackrel{\circ}{\stackrel{\circ}{0}}$ & $\frac{\circ}{\sin }$ & 总 & 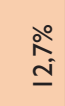 & $\begin{array}{l}\stackrel{\circ}{2} \\
\infty\end{array}$ \\
\hline \multirow{10}{*}{ 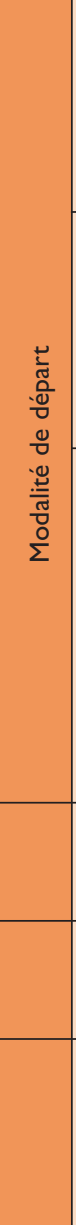 } & & a & & & $\bar{o}_{v^{\prime}}$ & & & & & $\begin{array}{l}\bar{o} \\
v^{\prime}\end{array}$ & & & $\begin{array}{l}n \\
\stackrel{n}{v} \\
\mathrm{v}\end{array}$ & 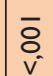 \\
\hline & & $=$ & $\cong$ & 岂 & के & 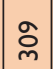 & $\underline{E}$ & $\stackrel{\infty}{\circ}$ & in & $\stackrel{\Re}{\wedge}$ & $\overline{\underline{ }}$ & $\bar{\infty}$ & กี & శ్ల \\
\hline & 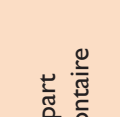 & 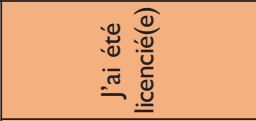 & $\stackrel{\text { ळo }}{-}$ & $\stackrel{\circ}{\stackrel{2}{\circ}}$ & 유 & 号 & $\stackrel{\circ}{=}$ & ڤે̀ & ○े & $\stackrel{\circ}{\mathrm{i}}$ & $\begin{array}{l}\text { @o } \\
0 \\
10\end{array}$ & $\frac{\circ}{\underline{0}}$ & よ̊ & $\stackrel{\stackrel{\circ}{m}}{\stackrel{\sim}{\sim}}$ \\
\hline & ב. & 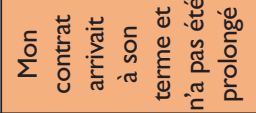 & 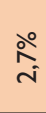 & 产 & 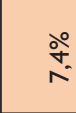 & $\frac{\circ}{0}$ & ○̊우 & خे & ○े & $\begin{array}{l}\stackrel{\circ}{\dot{े}} \\
\stackrel{0}{\dot{c}}\end{array}$ & $\stackrel{\circ}{\vec{\sigma}}$ & $\mid$ & よ̊ & $\begin{array}{l}\stackrel{\circ}{\circ} \\
\infty\end{array}$ \\
\hline & \multirow{3}{*}{ 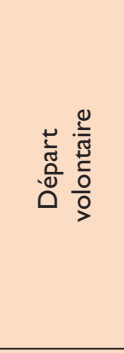 } & 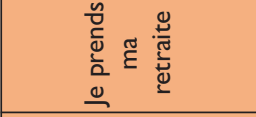 & ○े & 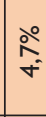 & ஓ̊ & \begin{tabular}{|l}
$\stackrel{\circ}{0}$ \\
0 \\
0
\end{tabular} & ⿳亠丷厂 & $\underset{\infty}{\stackrel{0}{\circ}}$ & 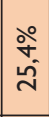 & $\frac{\stackrel{\circ}{\circ}}{\underline{\alpha^{\circ}}}$ & 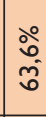 & $\mid$ & $\begin{array}{l}\stackrel{\circ}{\circ} \\
\stackrel{\circ}{q}\end{array}$ & $\begin{array}{l}\text { ڤे } \\
\text { å }\end{array}$ \\
\hline & & 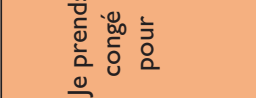 & 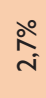 & ○े & 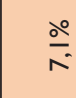 & $\frac{\frac{q}{2}}{\frac{q}{2}}$ & 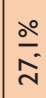 & $\begin{array}{l}\stackrel{2}{2} \\
\hat{0} \\
0\end{array}$ & 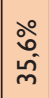 & 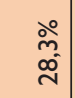 & 今े & $\mid$ & ํํํ & $\begin{array}{l}\stackrel{\circ}{0} \\
\infty \\
\infty \\
\infty\end{array}$ \\
\hline & & 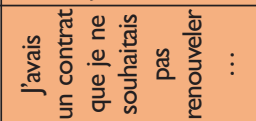 & 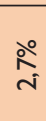 & ㅇํㄹ & ذْ & $\mid \begin{array}{c}\stackrel{\circ}{0} \\
\text { in }\end{array}$ & $\frac{\circ}{\omega^{\circ}}$ & $\stackrel{\stackrel{\circ}{+}}{=}$ & ○े & $\begin{array}{l}\stackrel{\circ}{2} \\
\text { o. }\end{array}$ & $\stackrel{2}{\stackrel{2}{2}}$ & 总 & ○ें & 突 \\
\hline & & 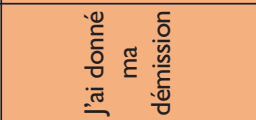 & & $\begin{array}{l}\text { ले } \\
\text { जैं }\end{array}$ & $\begin{array}{l}\text { ळ. } \\
\text { ळ. }\end{array}$ & \begin{tabular}{|l}
$\stackrel{\circ}{0}$ \\
$\dot{ \pm}$
\end{tabular} & $\begin{array}{l}\stackrel{\circ}{2} \\
\stackrel{\sim}{+}\end{array}$ & خે̀ & $\begin{array}{l}\text { oे } \\
\text { के }\end{array}$ & 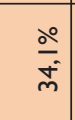 & $\begin{array}{l}\text { ळ } \\
\text { ळ. }\end{array}$ & 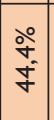 & $\begin{array}{l}\stackrel{\circ}{\circ} \\
\text { à }\end{array}$ & 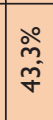 \\
\hline & & $z$ & $\stackrel{m}{=}$ & ٌे & $\underset{m}{\mathbb{N}}$ & $\underset{m}{\stackrel{a}{\infty}}$ & ¿ั & $\frac{m}{N}$ & $\underline{m}$ & $\bar{\Xi}$ & $\bar{\alpha}$ & $\frac{m}{N}$ & $\Xi$ & ஸี \\
\hline & & & 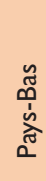 & $\begin{array}{l}\frac{0}{0} \\
\frac{\overrightarrow{0}}{b_{0}} \\
\frac{0}{0}\end{array}$ & 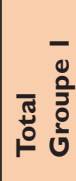 & 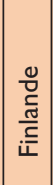 & 巳ัّ & 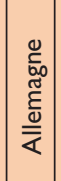 & $\frac{0}{\underline{\underline{\Xi}}}$ & 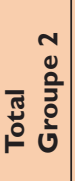 & $\begin{array}{l}0 \\
\frac{0}{6} \\
\frac{0}{0} \\
0\end{array}$ & $\begin{array}{l}. \frac{0}{\bar{z}} \\
\frac{2}{\omega} \\
\frac{0}{\omega}\end{array}$ & نे & 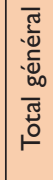 \\
\hline
\end{tabular}




\begin{tabular}{|c|c|c|c|c|c|c|c|}
\hline \multirow{3}{*}{ 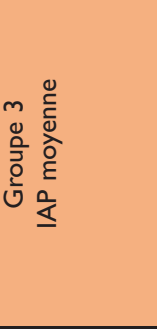 } & a & & \multicolumn{5}{|c|}{$\bar{o}$} \\
\hline & 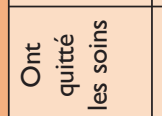 & $\mp$ & 承 & & 学 & 承 & đ̃ \\
\hline & 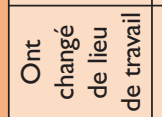 & $m$ & 品 & 昰 & $\begin{array}{l}\infty \\
\vdots \\
\vdots\end{array}$ & 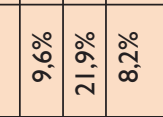 & $\stackrel{\wp}{œ}$ \\
\hline \multirow{3}{*}{ 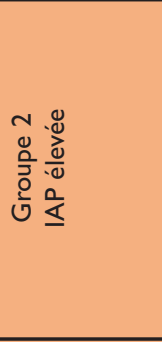 } & $\therefore$ & & \multicolumn{5}{|c|}{$\overline{\bar{g}}$} \\
\hline & 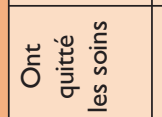 & $\underline{\Omega}$ & 总 & 商 & 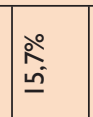 & 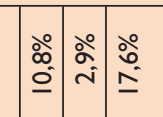 & 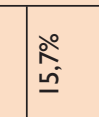 \\
\hline & 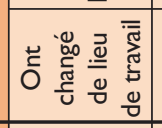 & $\overline{\bar{\rho}}$ & 然 & 品 & 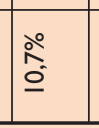 & 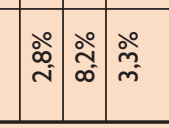 & 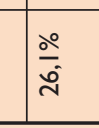 \\
\hline \multirow{3}{*}{ 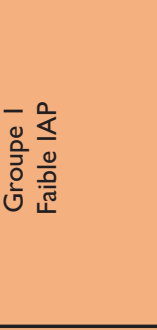 } & 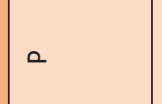 & & \multicolumn{5}{|c|}{$\bar{g}$} \\
\hline & 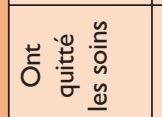 & 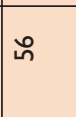 & 赵 & $\stackrel{\circ}{\stackrel{一}{\circ}}$ & 总 & 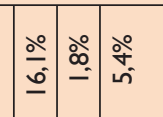 & 总 \\
\hline & 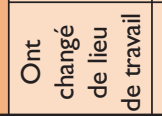 & $\tilde{\approx}$ & : & 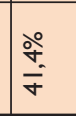 & 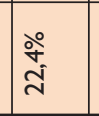 & 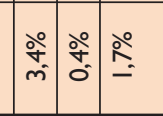 & 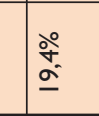 \\
\hline \multirow{4}{*}{ 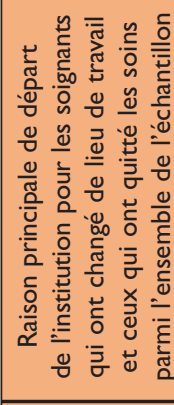 } & $\circ$ & . & \multicolumn{5}{|c|}{$\overline{\mathrm{g}} \mathrm{i}$} \\
\hline & 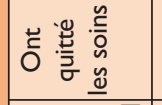 & $\stackrel{\alpha}{g}$ & 象 & $\frac{\circ}{\pi ٍ \pi}$ & 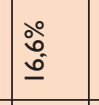 & 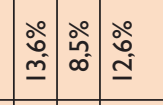 & 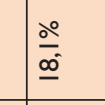 \\
\hline & 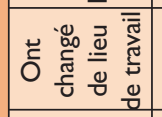 & $\mathscr{8}$ & 总 & 产 & ‡ْ & 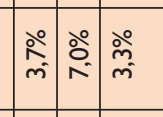 & 率 \\
\hline & $=$ & 足 & $\cong$ & 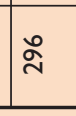 & $\underline{\underline{m}}$ & $\dddot{m}: \cong$ & $\underline{\underline{\infty}}$ \\
\hline \multirow{5}{*}{ 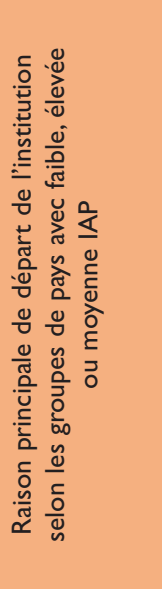 } & a & & \multicolumn{5}{|c|}{$\overline{\mathrm{o}}$} \\
\hline & 咞 & \& & 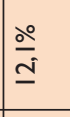 & 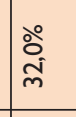 & 尘 & 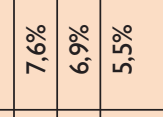 & 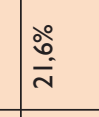 \\
\hline & 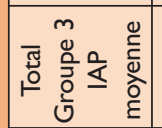 & $\cong$ & $\stackrel{\circ}{\%}$ & 㠃 & 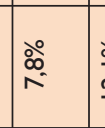 & 承 & $\dddot{\circ}$ \\
\hline & $\frac{2}{2}$ & $\overline{\mathrm{D}}$ & 离 & 产 & : & 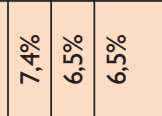 & 祐 \\
\hline & 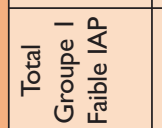 & 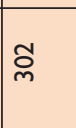 & 突 & 离 & 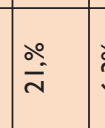 & 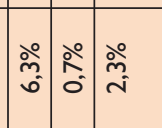 & 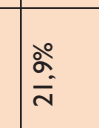 \\
\hline & & & & & & & \\
\hline
\end{tabular}




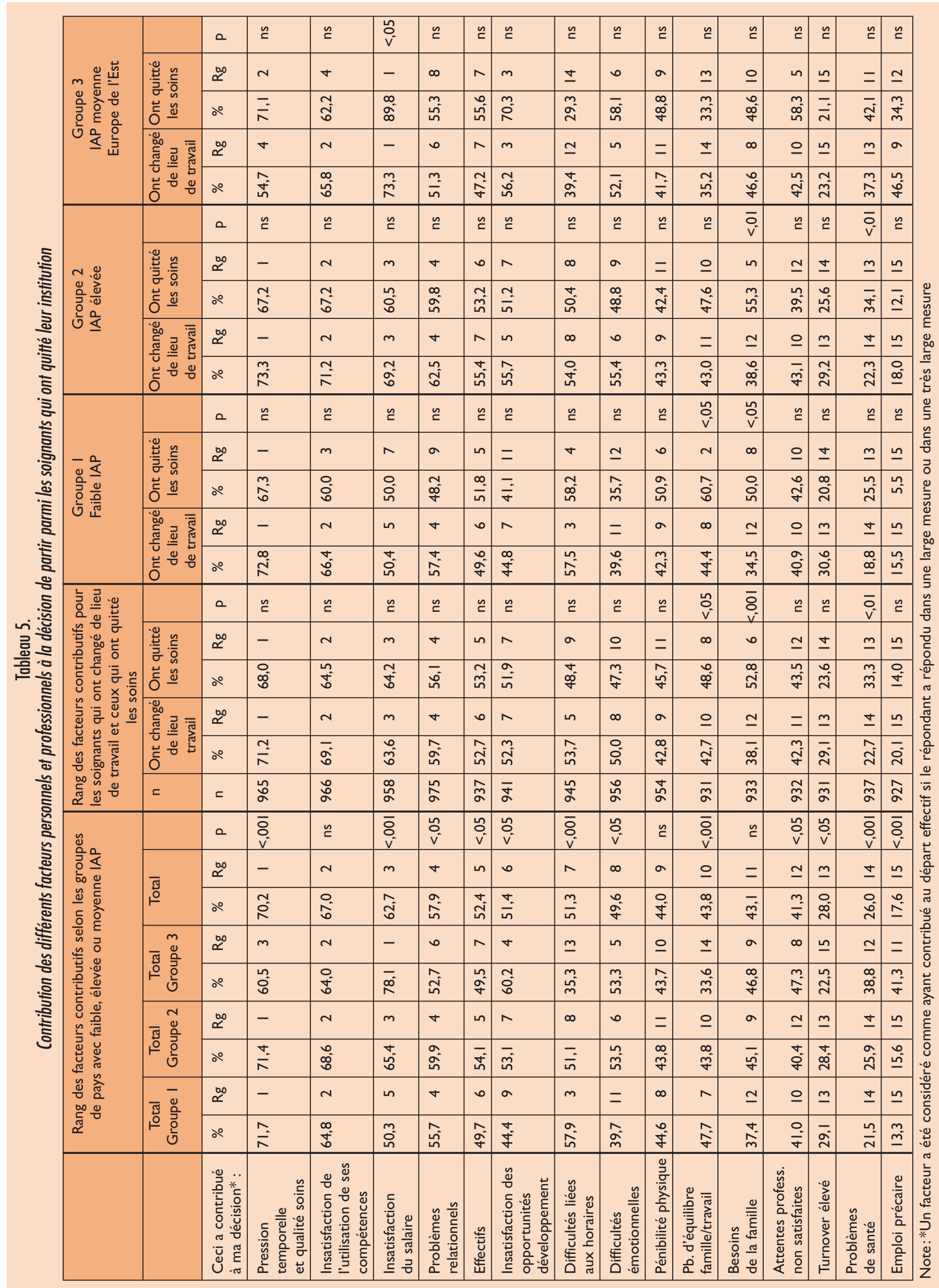

